\title{
The Ground State of the Nonlinear Sigma Model $\left(O(4)_{3+1}\right)$.
}

\author{
N.E. Ligterink ${ }^{* a}$, N.R. Walet ${ }^{a}$, and R.F. Bishop ${ }^{a}$ \\ a Department of Physics, UMIST, PO Box 88, \\ Manchester M60 1QD, \\ United Kingdom
}

We study the ground state of the Nonlinear Sigma Model in a hamiltonian framework as function of coupling constant, using the Coupled Cluster Method. We find a phase transition for finite coupling.

\section{INTRODUCTION}

The starting point for any investigation of a field theoretical model must be the vacuum. The ground state determines which type of fluctuations exist around this minimum, and thereby the low energy, or physical, states or particles. In many cases the bare vacuum can be the reference state for calculations. However, in some cases, such as with broken symmetries, the vacuum might be highly nontrivial. On top of that the ground state might depend strongly on the values of any physical constants that appear in the model field theory. There could, for instance, be a phase transition in the system.

We investigate the nonlinear sigma model as a model field theory to study the feasibility of using the coupled cluster method to study physical field theories. The nonlinear sigma model is believed to have a phase transition for a finite coupling constant[1]. We confirm this claim. The coupling constant in the nonlinear sigma model is proportional to the inverse square of the lattice spacing: and tells us in practice at what scale the ground state is oriented, and at what scale it is disoriented.

\section{THEORY}

The nonlinear sigma model is related to a number of physical systems. It was introduced to describe pion dynamics. The low-energy behaviou of pions and their isospin symmetry originate in the chiral symmetry of the quark degrees of free.

\footnotetext{
*Speaker
}

dom. The simplest nonlinear realization of chiral symmetry yields the lagrangian:

$$
\mathcal{L}=\frac{1}{4} \operatorname{Tr}\left[\partial_{\mu} U \partial^{\mu} U^{\dagger}\right]
$$

where $U$ is a unitary $2 \times 2$ matrix, and $U^{\dagger}$ its hermitian conjugate. The manifest symmetry is the left and right multiplication with a unitary matrix: $\left(L L^{\dagger}=1\right.$ and $\left.R R^{\dagger}=1\right)$

$$
\begin{aligned}
U & \rightarrow L U R, \\
U^{\dagger} & \rightarrow(L U R)^{\dagger}=R^{\dagger} U^{\dagger} L^{\dagger},
\end{aligned}
$$

under which the lagrangian is invariant.

In order to derive the associated hamiltonian we have to identify the proper, independent variables. We parametrize the unitary matrix by:

$$
U=n^{0}+i \vec{\sigma} \cdot \vec{n},
$$

where $\hat{n}=\left(n^{0}, \vec{n}\right)$ is a four-dimensional unit vector. Therefore the kinetic energy can be represented by the angular momentum operators in four dimensions. There are 6 angular momentum generators in 4 dimensions: $(i \neq j)$

$$
L_{i j}=-i\left(n^{i} \frac{\partial}{\partial n^{j}}-n^{j} \frac{\partial}{\partial n^{i}}\right) .
$$

The generators $L_{i j}$ preserve the constraint $|\hat{n}|=$ 1. Hence the hamiltonian density follows from this parametrization in terms of a field of unit vectors, $\hat{n}$ :

$$
\mathcal{H}=\frac{1}{2} \sum_{i<j} L_{i j}^{2}+\frac{1}{4} \operatorname{Tr}\left[\vec{\nabla} U \cdot \vec{\nabla} U^{\dagger}\right] .
$$

In order to deal with the potential part we have to discretize the space variable. If we use a lattice 
spacing $a$ we will find that the potential term at the point $i$ can be given the form

$$
\frac{1}{4} \operatorname{Tr}\left[\vec{\nabla} U_{i} \cdot \vec{\nabla} U_{i}^{\dagger}\right]=\sum_{<i, j>} \frac{1}{2 a^{2}}\left(1-\hat{n}_{i} \cdot \hat{n}_{j}\right)(6)
$$

where $\langle i, j\rangle$ indicates the sum over all nearestneighbor site pairs. Now the whole hamiltonian is expressed in term of the variables $\hat{n}_{i}$, which we will use for the rest of the paper.

We introduce the coupling constant, $\lambda \equiv$ $\left(2 a^{2}\right)^{-1}$. For large coupling constants the ground state will be an oriented state: all the vectors stand in the same direction. For $\lambda=0$ there is a free rotator in four dimensions at each lattice point. The different rotators do not feel their neighbors. The ground state is the product of S-states at each lattice point.

The strong-coupling perturbative expansion requires a basis consisting of the direct product of hyperspherical harmonics at each lattice point [2]. Instead we will use the Coupled Cluster Method and restrict ourselves to pairwise correlations between sites. Given a correlation between two lattice sites, it depends only on one variable, the angle between the two vectors: $\hat{n}_{i} \cdot \hat{n}_{j}$, the others variables being the overall orientation of the system. For the (almost) free system the natural choice for the correlation functions in the relative coordinate are the Gegenbauer polynomials. This is true for an arbitrary dimension of the sphere.

\subsection{COUPLED CLUSTER METHOD}

The Coupled Cluster Method (CCM) is a very direct way of calculating the ground state and the ground-state energy. The basis of the CCM is the parametrization of the bra and ket state with an exponential of all excitation operators $[3,4]$ :

$$
\begin{aligned}
& \langle\{\hat{n}\} \mid \psi\rangle=\mathrm{e}^{S} \\
& \langle\psi \mid\{\hat{n}\}\rangle=(1+\tilde{S}) \mathrm{e}^{-S} .
\end{aligned}
$$

The functions $S$ and $\tilde{S}$ are approximated as the sum of all pairwise correlations:

$$
\begin{aligned}
S & =\sum_{i, j} \sum_{\vec{m}} a_{|\vec{m}|, j} S_{j}\left(\hat{n}_{i} \cdot \hat{n}_{i+\vec{m}}\right), \\
S & =\sum_{i, j} \sum_{\vec{m}} b_{|\vec{m}|, j} S_{j}\left(\hat{n}_{i} \cdot \hat{n}_{i+\vec{m}}\right),
\end{aligned}
$$

where $i$ and $i+\vec{m}$ run over all lattice sites, and $j$ runs over a complete set of basis functions. The dependence on $|\vec{m}|$ only indicates that for all pairs of lattice points which are unique up to lattice symmetries (translations, rotations, and reflections) we use the same correlation function.

This parametrization takes proper care of independent excitations. For a number of systems CCM gives the best available results for groundstate energies. However, most of these systems are many-body systems and formulated in terms of operators. Field theoretical models are more easily formulated in terms of coordinate expectation values. Hence, the excitation operator will amount to multiplication with a function, and one excitation operator does not necessarily step through the whole Hilbert space.

Basically, since the state is normalized we only need one functional to derive most results:

$$
I[\vec{a}, \vec{b}]=\left\langle(1+\tilde{S}) \mathrm{e}^{-S} H \mathrm{e}^{S}\right\rangle,
$$

where $\vec{a}$ and $\vec{b}$ are the coefficients of the ket and bra state parametrization (eq. 8). If $\mathrm{e}^{S}$ is an eigenstate of the Hamiltonian, $\mathrm{e}^{-S} \mathrm{He}^{S}$ must be equal to $E_{g s}$ and the variation with respect to $\tilde{S}$, which are orthogonal to the reference state (the constant function in this case), must be zero:

$$
\frac{\delta I[\vec{a}, \vec{b}]}{\delta \vec{b}}=0
$$

The $\vec{a}$, which are a solution to these equations, lead immediately to the ground-state energy:

$$
E_{g s}=I[\vec{a}, 0] \text {. }
$$

This is all we need to find the ground state in the CCM. We will restrict ourselves to the SUB2 approximation, i.e., we allow only for two-body correlations. In this approximation the ground state is a product of functions of one relative angle only.

\section{EXCITATIONS}

Since the nonlinear sigma model describes pion dynamics, the excitations in the strongcoupling limit are associated with different particles. However, in the weak-coupling limit (disoriented state) the signatures of the excitations 
are not so clear. In the free case the lowest collective excitation is the sum of lowest free rotator excitations. We track this state to stronger couplings using an excitation operator. In order to estimate the energy gap between the groundstate energy and this excitation we calculate the commutator of the hamiltonian and the excitation operator. This excitation operator is the exact operator which put the system in the first excited state in the free case. Due to the variational form of the equations in the CCM we can calculate the energy gap in this approximation analytically, knowing the properties of the ground state. The excitation operator equals a specific combination of the correlation functions that appear in the bra and ket state.

The excitation operator:

$$
X=\sum_{<i, j>} \hat{n}_{i} \cdot \hat{n}_{j}
$$

is inserted in the expectation value of the hamiltonian using Feynman's technique, The energy gap is given by:

$$
\Delta E=-\frac{1}{2} \frac{\left\langle 0\left|(1+\tilde{S}) \mathrm{e}^{-S}[[H, X], X] \mathrm{e}^{S}\right| 0\right\rangle}{\left\langle 0\left|(1+\tilde{S}) \mathrm{e}^{-S} X^{2} \mathrm{e}^{S}\right| 0\right\rangle}
$$

where the bra and the ket state are those found in the calculation of the ground state.

\section{RESULTS}

The results depend on the order of approximation. We need to truncate both the number of correlation functions taken into account and how many basis functions are used for each retained correlation function. Generally the results depend more critically on the former than on the latter. Close to the phase transition point, observed in Fig 1, all the correlation functions are still smooth and not very large. They are very well approximated with as few as three basis functions. We allow all correlation functions within a cube and find that we already obtained the limit point to an accuracy of $\sim 2 \%$ with a cube of size 2 , which leads to 9 correlation functions.

The physical branch, which starts at the constant function at $\lambda=0$, bends downwards at the

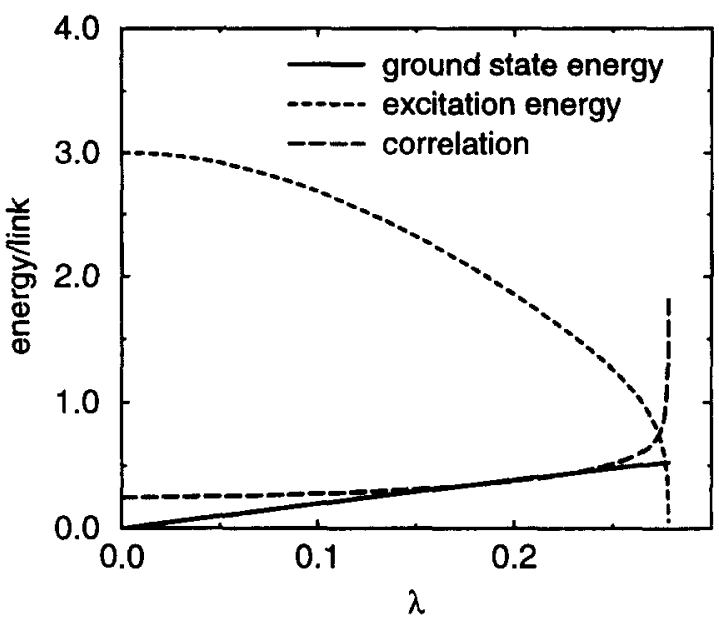

Figure 1. The ground-state energy and the energy gap. The extrapolated end point is $\lambda=0.2679(5)$. The calculation of the excitation energy becomes unstable at the phase transition point. The correlation is the expectation value of $X^{2}$ (see text).

phase transition point, and backtracks the physical branch at a slightly lower energy. At the highest-order approximation, with all correlations retained in a cube of size 4 , the two solutions are almost indistinguishable.

The vanishing energy gap between the ground state and the first excited state is a good indication of a phase transition at the end point. We aim both to investigate this further and to extend our results to other dimensions for comparison with other hamiltonian approaches [2].

\section{REFERENCES}

1. S.A. Chin (private communication).

2. C.J. Hamer, J.B. Kogut, and L. Susskind, Phys. Rev. D 19 (1979) 3091, G.E. Sobelman, Phys. Rev. B 24 (1981) 1493.

3. S.J. Baker, R.F. Bishop, and N.J. Davidson, Phys. Rev. D 53 (1996) 2610.

4. S.J. Baker, R.F. Bishop, and N.J. Davidson, Nucl. Phys. B (Proc. Supp.) 53 (1997) 834. 